\title{
O MAPA DO TERRENO DO APOIO MATRICIAL: UMA REVISÃO DIALÓGICA DA LITERATURA
}

\author{
Renata Laureano da Silva1 e Maria Auxiliadora Teixeira Ribeiro2 \\ 1Centro Universitário Tiradentes de Alagoas, Brasil. laureano.renata1@gmail.com \\ 2Instituto de Psicologia da Universidade Federal de Alagoas, Brasil. maria.ribeiro@ip.ufal.br
}

\begin{abstract}
Resumo. O Apoio Matricial surgiu ao constatar-se que a reforma psiquiátrica não pode avançar se a atenção básica não for incorporada ao processo. Este é definido como um arranjo organizacional que objetiva aumentar a resolubilidade das ações de saúde, por meio de equipes multidisciplinares e por uma conexão em rede. $\mathrm{O}$ que tem sido pesquisado sobre esse arranjo, objetiva-se relatar neste trabalho, por meio do mapeamento da literatura científica, acessada em banco de dados BIREME. A análise e leitura desse material foram norteadas pelas ideias dos pesquisadores Montuori e Walker, que defendem uma forma inovadora de revisão da literatura, denominada revisão dialógica da literatura. Apresentamos os critérios utilizados para a seleção de material, bem como os locais de estudo, a área de conhecimento dos autores e o foco de discussão das pesquisas, organizando, assim, um panorama geral dos estudos sobre Apoio Matricial. Oitenta e três estudos foram analisados e estabelecemos como ponto de diálogo as potencialidades do Apoio Matricial, as quais foram citadas pelos autores pesquisados como uma Educação Permanente, sendo que este arranjo permite uma forma de trabalho mais resolutiva. A contribuição deste estudo para as pesquisas qualitativas está na forma de fazer e apresentar a revisão da literatura.
\end{abstract}

Palavras-chave: Apoio Matricial; Revisão Dialógica de Literatura; Reforma Psiquiátrica.

\section{MAP OF MATRIX SUPPORT GROUND: A DIALOGICAL REVIEW OF THE LITERATURE}

\begin{abstract}
Matrix support came to be from the finding that a psychiatric reform cannot advance if basic care is not incorporated in the process. This is defined as an organizational arrangement to increase the solvability of healthcare actions, through multidisciplinary teams and a network connection. In this context, we will report the mapping of the scientific literature about this arrangement, accessed through research in BIREME's database. The analysis and reading of this material were guided by the ideas of researchers Montuori and Walker, who defend an innovative way of literature reviewing, named dialogical review of the literature. We presented the criteria used for selecting the material, as well as the study location, the area of knowledge of the authors and the focus of discussion of each research, as such organizing a general view of the studies related to matrix support. Eighty-three studies were analysed and it has been established as a dialogue point the potential of matrix support, which have been quoted by the authors researched like Permanent Education and this arrangement allows a more resolutive way of working. This is conceived as a creative and constructive process, in which there is a dialogue with the researchers who study matrix support. This study has contributed to present another way of making and presenting the literature review on qualitative research.
\end{abstract}

Keywords: Matrix Support; Dialogic Literature Review; Psychiatric Reform.

\section{INTRODUÇÃO}

Para iniciarmos a reflexão sobre o Apoio Matricial (AM), localizamos o movimento da Reforma Psiquiátrica no Brasil como um dos marcos sócio-históricos que permitiu a articulação entre Saúde Mental e Atenção Básica, propiciando, consequentemente, um campo para o debate sobre o matriciamento. 
Esse movimento faz uma crítica ao modelo hospitalocêntrico pregresso de cuidados às pessoas em sofrimento psíquico. Propõe que os manicômios e hospitais psiquiátricos sejam substituídos por dispositivos que possibilitem o tratamento e restabeleçam os laços sociais e familiares daqueles que apresentam transtorno mental.

$\mathrm{Na}$ busca de concretizar essa nova proposta, torna-se fundamental a articulação entre os serviços de saúde mental e atenção básica para a rede de saúde, visto que o foco sai apenas do processo doença e cura, e a saúde mental passa a ser considerada dentro da saúde coletiva como um processo psicossocial complexo.

Podemos compreender que o Apoio Matricial surgiu por meio da constatação de que a reforma psiquiátrica não poderia avançar se a atenção básica não fosse incorporada ao processo. Não é produtivo concentrar esforços somente na rede substitutiva, porém, é preciso abrir a rede de cuidados em saúde mental para todos os níveis de assistência, em especial, para a atenção primária (Dimenstein, Severo, Brito, Pimenta, Medeiros, \& Bezerra, 2009).

É nesse cenário que surge a proposta de que o Apoio Matricial seja definido como um arranjo organizacional, visando assegurar retaguarda especializada a equipes e profissionais nos serviços de saúde. Constitui-se como uma metodologia de trabalho complementar àquela prevista em sistemas hierarquizados, a saber: mecanismos de referência e contrarreferência, protocolos e centros de regulação. O AM pretende oferecer tanto retaguarda assistencial quanto suporte técnico-pedagógico às equipes de referência (Campos \& Domitti, 2007).

Ao analisar o termo Apoio Matricial, os autores indicam que o primeiro - Apoio -, nesse modelo organizacional, sugere uma metodologia para ordenar essa relação entre referência e especialista, com base em procedimentos dialógicos e não mais na autoridade e nas relações verticais. Já o segundo termo - Matricial - pode ter vários sentidos: em sua origem latina, significa o lugar onde se geram e se criam coisas, mas, aqui, é utilizado no sentido de sugerir que os profissionais de referência e os especialistas mantenham uma relação horizontal e não apenas vertical, como recomenda a tradição dos sistemas de saúde (Campos \& Domitti, 2007).

O Apoio Matricial e a equipe de referência, juntos, objetivam ampliar as possibilidades de realizar-se clínica ampliada e integração dialógica entre distintas especialidades e 
profissões. Portanto, são arranjos organizacionais e uma metodologia para a gestão do trabalho em saúde que pretendem assegurar maior eficácia e eficiência, mas também investir na construção de uma autonomia dos usuários (Campos \& Domitti, 2007).

As primeiras experiências de Apoio Matricial ocorreram no SUS de Campinas-SP, a partir de 1989 (Figueiredo \& Onocko-Campos, 2009). Diante dessas vivências, o Ministério da Saúde propôs a estratégia do Apoio Matricial (AM) para facilitar o direcionamento dos fluxos na rede, promovendo uma articulação entre os equipamentos de saúde mental e as Unidades Básicas de Saúde (Dimenstein et al., 2009).

Em 2015, deu-se início o desenvolvimento de projetos, que visavam a implantação do AM, na cidade de Maceió (Alagoas), município em que as pesquisadoras vivem, incentivando a realização de uma pesquisa mais ampla sobre as possibilidades de sua implementação.

A princípio, buscou-se conhecer o que tem sido produzido a respeito desse arranjo, por meio do mapeamento da literatura científica, acessada do banco de dados BIREME, identificando quais discursos permeavam as produções científicas sobre o Apoio Matricial.

Portanto, esse primeiro momento é aqui relatado, no qual conhecemos as produções científicas nacionais que abordavam a temática do Apoio Matricial, em que regiões, áreas de conhecimento e periódicos eram produzidas. Posteriormente, elegemos analisar as potencialidades discutidas por essas produções.

Essa análise foi norteada por uma revisão dialógica (Walker, 2015) da literatura. Salientamos que não encontramos estudos que abordassem Apoio Matricial utilizando o referencial teórico por nós adotado.

Este trabalho é um recorte da dissertação da primeira autora, orientada pela segunda, intitulada "As Versões de Apoio Matricial por um Grupo de Profissionais de Saúde", defendida em 2017.

\section{METODOLOGIA}

Em nosso percurso de formação acadêmica, estudamos diversos textos e abordagens sobre Psicologia e o método científico. Nesse vasto catálogo de opções, alguns deles são apenas mais uma leitura obrigatória, já, outros, nos marcam e nos acompanham por todo nosso caminho profissional, com reverberações por quaisquer rotas que trilhemos. Esses textos, 
ditos coloquialmente por nós como marcantes, nos fazem problematizar aquilo que tínhamos como certo e familiar e, especialmente, refletir sobre nossos modos de atuação/pesquisar. Tal efeito aconteceu com os escritos de Montuori (2005) e Walker (2015), que discutem a revisão da literatura no processo científico.

Concebemos que esta é uma etapa inerente a qualquer processo de pesquisa. Geralmente, é realizada e descrita de maneira semelhante pelos pesquisadores e não se constitui como um dos passos mais cativantes do empreendimento científico. No entanto, esses dois autores nos fizeram compreender a revisão de uma maneira totalmente inovadora e instigante.

A revisão da literatura defendida por Montuori (2005) abre uma oportunidade para pesquisas criativas, um diálogo com a comunidade científica e aqueles que fazem parte dela. Portanto, o revisor é um participante ativo na construção desse diálogo e não meramente um espectador que visa reproduzir da melhor maneira possível trabalhos e temas relevantes. $A$ revisão da literatura não pode ser exaustiva e reprodutiva. É o mapa do terreno e não o terreno em si. O pesquisador, nesse sentido, apresenta uma interpretação dos estudos em questão, que nos falam tanto do próprio pesquisador quanto dos estudos revisados.

Inspirada pelas ideias de Montuori (2005), a pesquisadora Walker (2015) também reflete sobre o processo de revisão da literatura. Para ela, essa etapa pode criar um conhecimento transformador. Ao realizar uma revisão, a autora busca expor o contexto relacional dos textos. Logo, monta conexões, explora os padrões e interações relacionais desses, como se os textos conversassem e debatessem de forma respeitosa entre si. Desse modo, busca estabelecer um diálogo entre os textos e consigo mesma.

Instigadas por essas ideias, procuramos traçar, neste trabalho, uma revisão dialógica da literatura técnico-científica sobre Apoio Matricial, concebendo esse passo como um processo criativo e construtivo, a fim de realizá-la não como algo distante de nós e, sim, como uma comunidade vivente permeada por histórias, paixões, controvérsias e alianças. Pontuamos que nesse tipo de revisão traçamos um panorama das produções e dialogamos com os temas investigados. 


\subsection{Levantamento na base de dados}

A pesquisa em base de dados possibilita nossa compreensão inicial sobre o campo-tema pesquisado e o acesso a materiais produzidos, em espaços e tempos distintos daqueles em que se encontra o pesquisador. Além disso, agrupa as informações de uma forma organizada e leva-nos a explorar os modos pelos quais os assuntos se tornam relevantes para a ciência, acessá-los e discuti-los criticamente. Esse processo não é meramente técnico e não há neutralidade em sua construção, mas se constitui por aspectos históricos, éticos e políticos (Ribeiro, Martins, \& Lima, 2015).

Campo-tema é compreendido como o complexo de redes de sentidos que se interconectam, um espaço criado, herdado ou incorporado pelo pesquisador ou pesquisadora e negociado na medida em que busca se inserir nas suas teias de ação. "Quando fazemos o que nós chamamos de pesquisa de campo, nós não estamos "indo" ao campo. Já estamos no campo, porque já estamos no tema" (Spink, 2004).

Para a revisão da literatura, buscamos na base de dados Bireme (acessada no dia 31 de maio de 2015) estudos relacionados ao Apoio Matricial. Os critérios que delimitaram e orientaram a seleção do material foram: I - Parâmetro temático: produções que abordassem ou pesquisassem sobre o Apoio Matricial; II - Parâmetro linguístico: estudos publicados em língua portuguesa; III - Principais fontes: periódicos; IV - Parâmetro cronológico de publicação: produções publicadas no período entre 1990 a 2015. Utilizamos o descritor "apoio matricial".

A delimitação à língua portuguesa deveu-se ao fato de o AM ser uma proposta exclusivamente brasileira. Para o parâmetro cronológico, incluímos a década de 1990, visto que as primeiras experiências em matriciamento aconteceram naquele ano.

Para a seleção das publicações, lemos cada título e resumo. Excluímos textos que não possuíam a temática do Apoio Matricial no cerne da discussão. Com as produções selecionadas realizamos, primeiramente, a leitura de cada título e resumo. Para a leitura na íntegra dos artigos, definimos as informações a serem extraídas dos trabalhos revisados, como objetivos, metodologia e principais conclusões. Estas informações eram catalogadas em fichas de leitura.

Nessas fichas, caracterizamos os aspectos avaliados como importantes para refletirmos sobre a temática do Apoio Matricial. Levamos em consideração os aspectos já citados, 
como os locais de estudo, a área de conhecimento dos autores e o foco de discussão das pesquisas para a construção do panorama geral. Estes dados foram quantificados em tabelas e serão apresentadas e discutidas a seguir.

Além disso, a análise das publicações selecionadas permitiu a identificação da maneira como os pesquisadores definiam o Apoio Matricial, quais termos, expressões e vocábulos utilizavam para conceituar esse arranjo e, principalmente, favoreceu a compreensão sobre as potencialidades e dificuldades do matriciamento. Todavia, enfocaremos as potencialidades deste.

Por fim, pontuamos que utilizaremos os termos apoio matricial e matriciamento concomitantemente, visto que são sinônimos.

\section{RESULTADOS E DISCUSSÕES}

De um total de 130 (cento e trinta) resultados obtidos com o levantamento bibliográfico, após a exclusão de artigos repetidos, bem como daqueles que não estavam disponíveis com texto completo para acesso (ou ainda, não se relacionavam com a temática e os critérios de nosso estudo mencionados anteriormente), obtivemos o quantitativo final de 68 (sessenta e oito) produções para análise. Desse total, 5 (cinco) eram dissertações, 3 (três) teses e uma cartilha produzida pelo Ministério da Saúde que também foi analisada.

No dia 14 de junho de 16, realizamos uma segunda pesquisa, na mesma base de dados, com o objetivo de incluir artigos mais recentes e que não apareceram na primeira busca. Seguimos os critérios supracitados, apenas incluindo o ano de 2016 no parâmetro cronológico. Nesse novo levantamento foram identificados mais 15 (quinze estudos), totalizando 83 (oitenta e três) trabalhos.

Nas produções analisadas, caracterizamos os aspectos avaliados como importantes para refletirmos sobre a temática do Apoio Matricial. Realizamos um diálogo com números, mas sem cair na falácia quantitativa, na qual os números são dados neutros, fidedignos de uma realidade ou verdade sobre o fenômeno estudado. O primeiro deles consiste em sinalizar as regiões brasileiras em que esses estudos foram feitos. A Tabela 1 apresenta a quantidade em ordem decrescente de estudos nas respectivas regiões. As pesquisas que apresentaram discussões teórico-conceituais identificadas como revisões de literatura não foram contabilizadas, visto que não possuíam um local de estudo delimitado; portanto, só 
consideramos as pesquisas empíricas. Por conseguinte, a Tabela 1 apresenta um total de 60 produções.

Tabela 1. Número de produções em ordem decrescente classificadas a partir das regiões brasileiras. Maceió, 2017.

\begin{tabular}{|c|c|}
\hline Regiões Brasileiras & Número de Produções \\
\hline Sudeste & 28 \\
\hline Nordeste & 21 \\
\hline Sul & 8 \\
\hline Centro-Oeste & 2 \\
\hline Norte & 1 \\
\hline
\end{tabular}

$\mathrm{Na}$ Tabela 1, notamos que a maioria dos artigos foram produzidos no Sudeste, tendo predominância aqueles oriundos do estado de São Paulo, especialmente Campinas, munícipio pioneiro na implementação e pesquisa sobre o Matriciamento e que recebe constantes investimentos em equipamentos substitutivos, como: Centros de Atenção Psicossociais (CAPS), Serviços Residenciais Terapêuticos (SRT), Centros de Convivência, Enfermarias de psiquiatria em Hospital Geral e Serviços de Geração de Renda (Ballarin, Blanes, \& Ferigato, 2012). Há também, nesse município, uma variedade de categorias profissionais e de locais de trabalho, a partir dos quais se realiza o Apoio Matricial que se expande para outros contextos, não só a Saúde Mental.

Uma pesquisa em todos os estados brasileiros (Fonseca Sobrinho et al., 2014) com as equipes de Saúde da Família indica que apenas no Sudeste as equipes de ESF avaliaram um alto grau de apoio. Na distribuição por estados, São Paulo, Rio de Janeiro e Santa Catarina apresentaram as maiores porcentagens de ações de apoio (Fonseca Sobrinho et al., 2014).

Em relação à região Nordeste, destacam-se os estudos oriundos do estado do Ceará. Os pesquisadores Bonfim, Bastos, Góis e Tofóli (2013) sinalizam as possíveis causas dessa crescente produção ao afirmarem que, a partir de 2006, as áreas de saúde mental e a atenção primária foram prioritárias em investimentos, o que proporcionou um processo gradual de implantação do AM em Saúde Mental, especialmente em Fortaleza.

Apontamos apenas uma pesquisa que abarca todos os estados brasileiros (Fonseca Sobrinho et al., 2014) e um estudo (Lacerda e Silva, Dias, Pessoa, Fernandes, \& Gomes, 2013) enfocando três regiões brasileiras: Norte, Nordeste e Centro-Oeste. 
Outro aspecto que abordamos foi a área de conhecimento dos pesquisadores, que pode ser visualizada na Tabela 2, no qual está relacionada também a quantidade de produções com a respectiva área de conhecimento dos autores.

Tabela 2. Número de produções em ordem decrescente classificadas a partir da Área de conhecimento dos autores. Maceió, 2017.

\begin{tabular}{|c|c|}
\hline Área de conhecimento & Número de Produções \\
\hline Multidisciplinar & 29 \\
\hline Saúde Pública & 17 \\
\hline Psicologia & 16 \\
\hline Saúde Coletiva & 9 \\
\hline Enfermagem & 7 \\
\hline Saúde Mental & 3 \\
\hline Medicina Preventiva e Social & 2 \\
\hline
\end{tabular}

Para definir a área de conhecimento, utilizamos as definições dos próprios autores nas produções. Quando não constavam, realizávamos uma pesquisa na Plataforma Lattes. A área multidisciplinar predominante nos estudos refere-se àquelas produções nas quais havia autores que pertenciam a mais de uma área de conhecimento. A produção de um conhecimento multiprofissional e interdisciplinar está condizente com a própria proposta de AM, que busca a construção coletiva e compartilhamento dos saberes. Além disso, as áreas de Saúde Pública e Saúde Coletiva também são campos interdisciplinares.

A análise das discussões apresentadas nos estudos nos levou a identificar dois focos que intitulamos: Discussões Conceituais e Operacionalidade do Conceito.

O primeiro caracteriza-se pelas produções que realizaram um estudo teórico elou crítico sobre o Apoio Matricial e, o segundo, refere-se às pesquisas empíricas sobre a temática, as quais foram classificadas de acordo com o foco da área da política pública estudada.

Sendo elas: Apoio Matricial e Atenção Básica; Apoio Matricial, Saúde Mental e Atenção Básica; Apoio Matricial e Saúde Mental; Apoio Matricial e Gestão; Apoio Matricial e Saúde do Trabalhador; Apoio Matricial, Atenção Básica e Gestão; Apoio Matricial, Saúde Mental, Atenção Básica e Gestão.

A Tabela 3 apresenta a quantidade de pesquisas em cada uma das categorias descritas. 
Tabela 3. Número de produções classificadas a partir dos focos de discussão das pesquisas. Maceió, 2017.

\begin{tabular}{|r|c|}
\hline Foco da Discussão & Número de Produções \\
\hline Discussão Conceitual & 20 \\
\hline Apoio Matricial e Atenção Básica & 21 \\
\hline Apoio Matricial, Saúde Mental e Atenção Básica & 15 \\
\hline Apoio Matricial e Saúde Mental & 14 \\
\hline Apoio Matricial e Gestão & 5 \\
\hline Apoio Matricial e Saúde do Trabalhador & 3 \\
\hline Apoio Matricial, Atenção Básica e Gestão & 2 \\
\hline Mpoio Matricial, Saúde Mental, Atenção Básica e & 1 \\
\hline
\end{tabular}

Esses critérios não se aplicam a duas produções, visto que apenas citam o AM como uma potencialidade para solucionar os problemas identificados nas pesquisas realizadas. $O$ matriciamento não é caracterizado como objeto de estudo dessas produções. Portanto, a Tabela 3 possui um total de 81 produções.

A grande parte das pesquisas selecionadas consiste em pesquisas empíricas sobre o Apoio Matricial, havendo um número expressivo daquelas que focalizam o AM e a Atenção Básica. Em termos gerais, há predominância da metodologia qualitativa, com a utilização de entrevistas como técnica de coleta de dados e os profissionais como informantes. A análise de dados é apenas citada, particularmente, como análise de conteúdo. Os pesquisadores não deixam claro quais passos, etapas e procedimentos realizaram para chegar às categorias temáticas ou aos resultados. Referendam as autoras, predominantemente Bardin ou Minayo, o que é insuficiente para dar visibilidade ao processo de análise dos resultados das pesquisas.

Alguns estudos se diferenciam ao levarem em conta as informações obtidas dos familiares e/ou usuários, não se atendo apenas às falas dos profissionais (Iglessias \& Avellar, 2014; Jorge, Vasconcelos, Neto, Gondim, \& Simões, 2014). Para compreender a prática do matriciamento, discutem a contribuição e participação dos usuários e seus familiares no processo de produção de conhecimento em relação ao Apoio Matricial (Quinderé, Jorge, Nogueira, Costa, \& Vasconcelos, 2013; Pinto et al., 2012; Jorge, Sousa, \& Franco, 2013; Onocko-Campos et al., 2011).

A partir da leitura dos resumos de todos os artigos e apoiadas no referencial teóricometodológico das práticas discursivas e produção de sentidos fundamentado na perspectiva do Construcionismo Social (Spink, 2004), procuramos compreender alguns dos aspectos do conhecimento construído sobre Apoio Matricial, dentre eles, as potencialidades em sua prática. Partimos de uma análise dos discursos científicos e as versões destes, na produção 
das práticas discursivas. Além disso, não encontramos estudos que abordem sobre a temática utilizando o referencial teórico por nós adotado.

Ao analisar os repertórios sobre o Apoio Matricial produzidos na literatura científica, realizamos uma reflexão sobre as potencialidades desse arranjo discutidas nos estudos.

Primeiramente, a Educação Permanente, incentivada por esse arranjo, é apontada como potencializadora na superação de vários desafios na rede de serviços de saúde (Minozzo, 2011; Ballarin et al., 2012; Bonfim et al., 2013; Fonseca Sobrinho et al., 2014; Jorge, Diniz, Lima, \& Penha, 2015; Fittipaldi, Romano, \& Barros, 2015). Nesses estudos, o matriciamento e a educação permanente são apontados como trabalhos associados.

A educação permanente foi pontuada como relevante por seu potencial de qualificação das práticas em saúde e de reorganização do processo de trabalho (Fittipaldi et al., 2015). Para os profissionais que colaboraram nas pesquisas, a educação permanente possibilita a discussão da clínica psicossocial, permitindo, assim, uma aproximação com o debate sobre os casos de saúde mental (Minozzo, 2011).

Além disso, o compartilhamento de saberes proporcionado pela Educação Permanente e Apoio Matricial permite a ampliação dos conhecimentos da equipe e sua capacidade de atendimento, propiciando uma aproximação não só entre seus membros, como de toda equipe com os usuários (Ballarin et al., 2012).

Outra potencialidade do AM discutida é que o fluxo da assistência se torna mais resolutivo devido à aproximação das equipes e articulação entre diferentes níveis de atenção (Bezerra \& Dimenstein, 2008; Dimenstein, Galvão, \& Severo, 2009; Quinderé et al., 2013; Hirdes \& Scarparo, 2013).

O Apoio Matricial possibilita distinguir as situações individuais e sociais, que podem ser acolhidas pela equipe local, daquelas demandas que necessitam de uma atenção especializada da saúde mental de acordo com o risco e a gravidade, além da necessidade de um acompanhamento por um serviço substitutivo (Dimenstein et al., 2009). Por meio do princípio da territorialidade, o AM interliga diferentes dispositivos com a articulação e o assessoramento da rede básica (Bezerra \& Dimenstein, 2008). 
Outra potencialidade apontada nos trabalhos consiste em que o AM fortalece o trabalho interdisciplinar (Brasil, 2004; Campos \& Domitti, 2007; Bezerra \& Dimenstein, 2008; Minozzo, 2011; Santos \& Lacaz, 2012; Quinderé et al., 2013).

O AM permite lidar com a saúde de uma forma ampliada e integrada, por meio de um saber generalista e interdisciplinar. Dessa forma, amplia o olhar dos profissionais da saúde mental, em virtude do conhecimento das equipes nas unidades básicas de saúde, em relação aos usuários, às famílias e ao território, propondo que os casos sejam de responsabilidade mútua (Bezerra \& Dimenstein, 2008).

Há um reconhecimento pelos estudos de que essa forma de trabalho é resolutiva (Quinderé et al., 2013; Santos \& Lacaz, 2012; Pinto et al., 2012; Galvão, 2012) e, consequentemente, aumenta a resolubilidade dos casos de saúde mental.

\section{CONCLUSÕES}

A Revisão de Literatura Dialógica contribuiu para apresentarmos uma forma de fazer e apresentar a revisão da literatura, na medida em que possibilita uma aproximação do pesquisador com o problema investigado nas pesquisas documentais, como um primeiro momento de uma pesquisa mais ampla sobre o tema de interesse.

Com este trabalho, não pretendemos afirmar essa possibilidade como sendo a correta, porém como um convite aos investigadores a realizarem estudos que explorem novos enfoques. No entanto, queremos destacar a importância da revisão da literatura dialógica nas pesquisas qualitativas, confrontando especialmente a ideia de que nesse tipo de pesquisa não seria necessário partir dessa etapa e/ou até mesmo evitá-la. Este posicionamento que podemos avaliar como retrógrado deve-se ao fato de que a pesquisa qualitativa esteve por muito tempo relacionada à ideia de exploração apenas de novos campos ou temas.

Salientamos que não encontramos trabalhos que abordem o Apoio Matricial utilizando o referencial teórico por nós adotado. Podemos concluir que este estudo permitiu o exame de materiais visando novas e/ou interpretações complementares.

Por meio da análise da produção científica, norteada por uma revisão dialógica da literatura, compreendemos que o fio condutor do trabalho de matriciamento é o compartilhamento de saberes, competências, responsabilidades e ações. O AM é um arranjo que busca mudar o 
cuidado pautado no modelo biomédico hegemônico, ao dissolver as fronteiras entre as disciplinas, o que não indica reduzir uma à outra, mas impulsionar em cada profissional de saúde a capacidade de uma prática integral e humanizada.

Em pesquisas sobre AM, mais recentes (Vendruscolo et al., 2020, Lima \& Gonçalves, 2020, Saraiva, Zepeda, \& Liria, 2020), a Educação Permanente e o matriciamento continuam sendo apontados como trabalhos associados e uma estatégia fundamental para a organização do cuidado psicossocial e trabalho em equipe.

Compreendemos que a metodologia proposta para a implantação do AM é complexa, acrescida da dificuldade em compatibilizar seus princípios com a prática vigente nos serviços de saúde. Constitui-se como um desafio, assim como a metodologia da revisão dialógica da literatura, por ambas se caracterizarem como um modelo que possibilita transpor as barreiras de um saber compartimentado e construir um saber integrado.

\section{REFERÊNCIAS}

Ballarin, M. L. G. S., Blanes, L. S., \& Ferigato, S. L. (2012). Apoio matricial: Um estudo sobre a perspectiva dos profissionais da saúde mental. Interface: Comunicação, Saúde e Educação, 16, 767-78.

Bezerra, E., \& Dimenstein, M. (2008). Os CAPS e o trabalho em rede: Tecendo o apoio matricial na atenção básica. Psicologia: Ciência e Profissão, 28, 632-645.

Bonfim, I. G., Bastos, E. N. E., Góis, C. W. L., \& Tófoli, L. F. (2013). Apoio matricial em saúde mental na atenção primária à saúde: Uma análise da produção científica e documental. Interface: Comunicação, Saúde e Educação, 17(45), 287-300.

Brasil (2004). Ministério da Saúde. Secretaria-Executiva. Núcleo Técnico da Política Nacional de Humanização. HumanizaSUS: Equipe de referência e apoio matricial / Ministério da Saúde, Secretaria-Executiva, Núcleo Técnico da Política Nacional de Humanização. Brasília: Ministério da Saúde.

Campos, G. W. S., \& Domitti, A. C. (2007). Apoio matricial e equipe de referência: Uma metodologia para gestão do trabalho interdisciplinar em saúde. Cad. Saúde Pública, 23(2), 399-407.

Dimenstein, M., Galvão, V. M., \& Severo, A. K. (2009). O apoio matricial na perspectiva de coordenadoras de equipes de Saúde da Família. Pesquisas e Práticas Psicossociais, 4(1).

Dimenstein, M., Severo, A. K., Brito, M., Pimenta, A. L., Medeiros, V., \& Bezerra, E. (2009). O apoio matricial em unidades de saúde da família: Experimentando inovações em saúde mental. Saúde Soc, 18(1), 63-74.

Figueiredo, M. D., \& Onocko-Campos R. (2009). Saúde Mental na atenção básica à saúde de Campinas, SP: Uma rede ou um emaranhado? Ciência \& Saúde Coletiva, 14(1), 129-138.

Fittipaldi, A. L. M., Romano, V. F., Barros, D. C. (2015). Nas entrelinhas do olhar: Apoio matricial e os profissionais da estratégia saúde da família. Saúde Debate, 39(104), 76-87.

Fonseca Sobrinho, D., Machado, A. T. G. M., Lima, A. M. L. D., Jorge, A. O., Reis, C. M. R., Abreu, D. M. X., \& Santos, A. F. (2014). Compreendendo o apoio matricial e o resultado da certificação de qualidade nas áreas de atenção à criança, mulher diabetes/hipertensão e saúde mental. Saúde Debate, 38, 83-93. 
Galvão, C. A. V. (2012). Saúde Mental e atenção primária: As implicações e desafios do apoio matricial em saúde mental na perspectiva da gestão em saúde (Dissertação de Mestrado). Escola Nacional de Saúde Pública Sergio Arouca, Rio de Janeiro, Brasil.

Iglesias, A., \& Avellar, L. Z. (2014). Apoio matricial: Um estudo bibliográfico. Ciência \& Saúde Coletiva, 19(9), 3791-3798.

Jorge, M. S., Diniz, A. M., Lima, L. L., \& Penha, J. C. (2015). Apoio matricial, projeto terapêutico singular e produção do cuidado em saúde mental. Texto Contexto Enfermagem, 24(1), 112-20.

Jorge, M. S. B., Vasconcelos, M. G. F., Neto, J. P. M., Gondim, L. G. F., \& Simões, E. C. P. (2014). Possibilidades e desafios do apoio matricial na atenção básica: Percepções dos profissionais. Psicologia: Teoria e Prática, 16(2), 63-74.

Jorge, M. S. B., Sousa, F. S. P., \& Franco, T. B. (2013). Apoio matricial: Dispositivo para resolução de casos clínicos de saúde mental na Atenção Primária à Saúde. Revista Brasileira de Enfermagem, 66(5), 738-44.

Lacerda e Silva, T., Dias, E. C., Pessoa, V. M., Fernandes, L. M. M., \& Gomes, E. M. (2013). Saúde do trabalhador na atenção primária: Percepções e práticas de equipes de Saúde da Família. Interface: Comunicação, Saúde e Educação, 18(49), 973-988.

Lima, M. C., \& Gonçalves, T. R. (2020). Apoio matricial como estratégia de ordenação do cuidado em saúde mental. Trab. Educ. Saúde, 18(1), 1-21.

Minozzo, F. (2011). Análise da implantação do apoio matricial em saúde mental entre CAPS e equipes de saúde da família: Trilhando caminhos possíveis (Dissertação de Mestrado). Universidade de Brasília, Brasília, Brasil.

Montuori, A. (2005). Literature review as creative inquiry: reframing scholarship as acreative process. Journal of Transformative Education, 3(4), 374-393.

Onocko-Campos, R., Gama, C. A., Ferrer, A. L., Santos, D. V. D., Stefanello, S., Trapé, T. L., \& Porto, K. (2011). Saúde mental na atenção primária à saúde: Estudo avaliativo em uma grande cidade brasileira. Ciência \& Saúde Coletiva, 16(12), 4643-4652.

Pinto, A. G. A., Jorge, M. S. B., Vasconcelos, M. G. F., Sampaio, J. J. C., Lima, G. P., Bastos, V. C., \& Sampaio, H. A. C. (2012). Apoio matricial como dispositivo do cuidado em saúde mental na atenção primária: Olhares múltiplos e dispositivos para resolubilidade. Ciência \& Saúde Coletiva, 17(3), 653-660.

Quinderé, P. H. D., Jorge, M. S. B., Nogueira, M. S. L., Costa, L. F. A., \& Vasconcelos, M. G. F (2013). Acessibilidade e resolubilidade da assistência em saúde mental: A experiência do apoio matricial. Ciência \& Saúde Coletiva, 18(7), 2157-2166.

Ribeiro, M. A. T., Martins M. H. M., \& Lima, J. M. (2015). A pesquisa em banco de dados: Como fazer? In Lang, C. E., Bernardes, J. F., Ribeiro, M. A. T. \&, Zanotti, S. V. (Orgs.) Metodologias: Pesquisas em saúde, clínica e práticas psicológicas (pp. 61-83). Maceió: Edufal.

Santos, A. P. L., \& Lacaz, F. A. C. (2012). Apoio matricial em saúde do trabalhador: Tecendo redes na atenção básica do SUS, o caso de Amparo/ SP. Ciência \& Saúde Coletiva, 17(5),1143-1150.

Saraiva, S. A. L., Zepeda, J., \& Liria, A. F. (2020). Componentes do apoio matricial e cuidados colaborativos em saúde mental: Uma revisão narrativa. Ciência \& Saúde Coletiva, 25(2):553-565.

Spink, M. J. (2004). Linguagem e produção de sentidos no cotidiano. Porto Alegre: EDIPUCRS.

Spink, P. K. (2003). Pesquisa de campo em psicologia social: Uma perspectiva pós-construcionista. Psicologia \& Sociedade, 15(2), 18-42.

Vendruscolo C., Trindade L. L., Metelski F. K., Vandresen L., Pires D. E. P, Tesser C. D., \& Martins M. M. F. P. S. (2020). Contribuições da educação permanente aos núcleos ampliados de saúde da família. Esc Anna Nery, 24(3), 1-8.

Walker, S. (2015). Literature reviews: generative and transformative textual conversations. Forum Qualitative Social Research, 16(3). 